\title{
Surgical treatment of undisplaced femoral neck fractures in the elderly
}

\author{
Lee Yih-Shiunn $\cdot$ Huang Chien-Rae $\cdot$ Liao Wen-Yun
}

Received: 3 July 2006 /Revised: 22 July 2006 / Accepted: 24 July 2006 / Published online: 11 October 2006

(C) Springer-Verlag 2006

\begin{abstract}
The study was a retrospective evaluation and comparison. Eighty-four elderly patients ( $>60$ years) with undisplaced intracapsular femoral neck fractures were treated with osteosynthesis with either dynamic hip screws (DHS) or multiple cannulated screws (MCS). The Singh index was used to evaluate bone quality. All patients were followed up retrospectively for at least 12 months. The clinical results were compared between the DHS and MCS groups. Both groups were similar in respect of injury mechanisms, mean Singh index, injury-surgery interval, gender and age (all $p$ values $\geq 0.29$ ). The MCS group had significantly smaller wound incisions, less haemoglobin level drops, lower blood transfusion rates and shorter hospital stays than the DHS group (all $p$ values $\leq 0.008$ ). However, the DHS group had a higher rate of overall success when compared to the MCS group $(97.5 \%$ versus $84.1 \%, p=0.04)$. In conclusion, although DHS fixation requires a larger skin incision and more soft tissue dissection, its use in elderly patients with osteoporosis is recommended due to simple, efficacy and high overall success rate.

Résumé Etude rétrospective, avec un suivi d'au moins 12 mois, de 84 patients âgés de plus de 60 ans avec une fracture intracapsulaire sans déplacement du col fémoral, traités par ostéosynthèse avec soit une vis-plaque dynamique (DHS) soit plusieurs vis canulées (MCS). L'index de Singh était utilisé pour évaluer la qualité osseuse. Les 2 groupes étaient similaires par le mécanisme traumatique, l'index de Singh, le délai de la chirurgie, le genre et l'âge. Le groupe MCS avait une plus courte incision, une moindre chute du niveau d'hémoglobine, un plus faible taux de transfusion et un plus
\end{abstract}

L. Yih-Shiunn $(\bowtie) \cdot H$. Chien-Rae $\cdot$ L. Wen-Yun

Taipei City Hospital, Heping Branch,

No. 33, Section 2, Chung-Hwa Rd,

Taipei 100, Taiwan

e-mail: dae10@tpech.gov.tw court séjour hospitalier que le groupe DHS. Cependant le groupe DHS avait un meilleur taux global de succès que le groupe MCS ( $97,5 \%$ versus $84,1 \%, p=0,04$ ). En conclusion, bien que la fixation par vis-plaque dynamique demande une plus grande dissection des parties molles, son utilisation chez les patients âgés et ostéoporotiques est recommandé en raison de son taux global de succès.

\section{Introduction}

Treatment of femoral neck fracture varies according to patient's age and fracture pattern $[9,13,20]$. For the undisplaced femoral neck fractures, rigid fixation with early mobilisation of patients is the standard of treatment $[2,6,8$, $15,19]$. Previously, multiple cannulated screws (MCS) or dynamic hip screws (DHS) have been commonly used to treat femoral neck fractures $[10-12,17,21,23,24]$. Osteosynthesis with MCS fixation is a less invasive technique, with less soft tissue stripping [6]. However, early loosening of the screws may occur if the lateral cortex is damaged from osteoporosis [9]. In contrast, DHS, which is a screw-plate system with fixed angles, can achieve more stable fixation in patients with osteoporosis. However, the disadvantages of DHS technique are larger skin incisions and more soft tissue dissection. This situation inspired us to retrospectively follow up and compare the clinical outcome of undisplaced femoral neck fractures that were treated with DHS or MCS.

\section{Materials and methods}

Between 1997 and 2003, 114 elderly, (> 60 years old) consecutive patients were surgically treated in our orthopaedic 
department for undisplaced and impacted femoral neck fractures. Inclusion criteria for this study were: (a) acute and intracapsular fractures; (b) all patients older than 60 years; (c) internal fixation with either MCS or a 3-hole DHS (before 2005, the shortest DHS side plate used at our hospital was 3-hole); (d) patients able to walk without any assistance before injury. Exclusion criteria for this study were: (a) basicervical fractures; (b) bilateral hip fractures; (c) pathological fractures; (d) patients who required intensive care or treatment in other departments; (e) previous ipsilateral hip fracture or surgery. There were 94 patients (DHS, $n=46$; MCS, $n=48$ ) who met the inclusion criteria. However, ten patients could not be followed up due to death (five cases) or relocation (five cases), and they were excluded. Eighty-four patients with an average age of 71.6 years were followed up for at least 12 months after discharge from the hospital and were included in this study. Eight surgeons were involved in our study. Four surgeons favoured DHS and the remaining four surgeons favoured MCS to treat undisplaced intracapsular fracture.

The 84 patients were divided into two groups, based on the method of treatment. The DHS group included 40 patients with an average age of 72.8 years. Thirty-three patients $(82.5 \%)$ suffered from simple falls. The MCS group included 44 patients with an average age of 70.6 years. Thirty-seven patients (84.1\%) suffered from simple falls (Table 1).

Distinction between undisplaced and displaced femoral neck fractures was made according to anteroposterior (AP), lateral and frog view radiographs. We reviewed the preoperative X-ray films of all the patients to record their bone quality, as defined by the Singh index [22]. The injury mechanism and preoperative demographics of each patient were recorded in detail during a retrospective chart review.

\section{Surgical techniques}

All the patients were placed on a radiolucent fracture table and their fractures were confirmed using fluoroscopy before surgery was initiated. In the DHS group, the standard procedures were performed $[9,24]$. A guide wire was inserted approximately $2 \mathrm{~cm}$ below the vastus lateralis ridge and a $135^{\circ}$ guide plate was placed close to the femoral shaft in a parallel position. As the guide wire was inserted, fluoroscopy was used to confirm satisfactory alignment, as judged on AP and lateral views. Then, a second guide wire was inserted temporarily before reaming to avoid proximal fragment rotation. The insertion of the second guide wire was approximately $1 \mathrm{~cm}$ above the first guide wire, in a parallel direction. After reaming, the lag screw was introduced. And then, all the guide wires were removed before insertion of the side plate. The operative procedure of MCS was standard and followed the three-point principle, with the insertion of three $6.5 \mathrm{~mm}$ (AO) cannulated screws [4]. One day after surgery, haemoglobin levels were checked; if haemoglobin was lower than $85 \mathrm{~g} / \mathrm{L}$ in association with increased heart rate or decreased blood pressure, blood transfusion was considered.

Plain films in the immediate postoperative period were reviewed for adequacy of fracture reduction and screw position for each patient by the all authors of the study during a retrospective review. Reduction was considered adequate when (a) there was no varus angulation, (b) posterior angulation was less than $5^{\circ}$ in the lateral view and (c) the proximal fragment was not inferior to the distal fragment in the AP view. Lag screw position of DHS was considered adequate when (a) the screw was inserted over the inferior calcar and in the lower half of the femoral head on the AP view, (b) the screw was inserted in the center or slightly in the dorsal part of the femoral head and through the central part of the femoral neck on the lateral view and (c) screw tip was within $5 \mathrm{~mm}$ from the subchondral bone but not penetrating the joint on either the AP or the lateral view. MCS position was considered adequate [15] when (a) screws were parallel (less than $5^{\circ}$ deviation), (b) all screws tips were within $5 \mathrm{~mm}$ form the subchondral bone but not penetrating the joint, (c) not all screws were in the cranial half of the femoral head and (d) not all screws were in the anterior half of the femoral head.

Partial weight bearing with crutches or walker assistance was routine for all patients for at least four weeks after discharge, and full weight bearing was permitted after six weeks, depending on individual clinical condition. Patients were reviewed at one, two, three, six, and 12 months after fracture. AP and lateral roentgenograms were taken for all patients at each follow-up appointment for evaluations of fracture healing, implant position, and the general condition of the hip joint. Radiographic healing was interpreted by the attending surgeon at each follow-up and was double confirmed by the all authors of this study during a retrospective review.

At the last follow-up, we evaluated the results using the Harris hip scoring system. In this system, both subjective and objective clinical data are included, with a maximum score of 100 points. The Student t-test, Chi-Square test with Yates' correction, and Fisher's exact test were used to compare the two groups. The statistic software SPSS 10.0 was used to analyse the data: $p$ values below 0.05 were considered significant.

\section{Results}

Forty of the original $46(87.0 \%)$ treated with DHS and 44 of the original $48(91.7 \%)$ treated with MCS had complete follow-up $(\mathrm{p}=0.34)$. Three out of $46(6.5 \%)$ patients treated 
with DHS and two out of $48(4.2 \%)$ patients treated with MCS died before the final follow-up ( $\mathrm{p}=0.48)$. The causes of death were not directly related to their hip fractures. The average period of follow-up was 33.5 months (range 1360 months) for the DHS group and 35.6 months (range 1268 months) for the MCS group $(p=0.40)$. Both the groups were similar in respect of injury mechanisms, mean Singh index, injury-surgery interval, gender, age and mean preoperative haemoglobin (all $p$ values $\geq 0.29$ ) (Table 1). Furthermore, if Singh index $\leq 3$ was defined as osteoporosis, the osteoporotic rate was $21 / 40(52.5 \%)$ in the DHS group and 22/44 (50\%) in the MCS group. The osteoporotic rate showed no significant difference (Fisher's exact test, $p=1.0$ ) between the groups.

The satisfactory reduction rates showed no significant difference $(p=1.0)$ between the two groups. The DHS group showed a tendency towards an increased rate of adequate screw position compared with the MCS group, although this was not statistically significant $(p=0.15)$. Thirty-six of $40(90 \%)$ DHS and 34 of $44(77.3 \%)$ MCS had adequate screw positions. All but two MCS fractures healed within six months. Differences in healing time and surgery time between the two groups were not significant $(p>0.2)$. The average incision length was significantly larger in the DHS group compared to the MCS group. The average haemoglobin level drop was significantly higher in the DHS group $(28.6 \pm 3.5 \mathrm{~g} / \mathrm{L})$ compared to the MCS group $(15.4 \pm 2.1 \mathrm{~g} / \mathrm{L})$. This resulted in a significantly higher blood transfusion rate in DHS group (25\%) compared to the MCS group (4.5\%). The average hospital stay was longer $(p<0.001)$ in the DHS group (10.6 \pm 1.92 ; range: $4-16$ days) than those in MCS group (6.2 \pm 1.52 ; range: $3-11$ days) (Table 2 ).

Table 1 Preoperative demographics and medical conditions in the 2 groups

\begin{tabular}{llll}
\hline & DHS & MCS & p value \\
\hline Simple fall (N) & 33 & 37 & 0.54 \\
Vehicular trauma (N) & 4 & 3 & 0.45 \\
Fall from a height (N) & 3 & 4 & 0.55 \\
Mean Singh index & 3.23 & 3.32 & 0.49 \\
Osteoporosis (N) & 21 & 22 & 1.0 \\
Injury-surgery interval (hours) & 33.8 & 36.9 & 0.29 \\
Gender (F/M) & $24 / 16$ & $25 / 19$ & 0.47 \\
Mean Age (years) & 72.8 & 70.6 & 0.38 \\
Mean body weight $(\mathrm{Kg})$ & 66.5 & 67.2 & 0.39 \\
Average stature $(\mathrm{cm})$ & 163.8 & 165.7 & 0.34 \\
Mean Preoperative $\mathrm{Hb}(\mathrm{g} / \mathrm{L})$ & 120.4 & 121.6 & 0.48 \\
\hline
\end{tabular}

DHS=dynamic hip screw

$\mathrm{MCS}=$ multiple cannulated screws

$\mathrm{N}=$ number of patients

$\mathrm{Hb}=$ haemoglobin
Table 2 Clinical results and hospital data in the 2 groups

\begin{tabular}{llll}
\hline & $\begin{array}{l}\text { DHS } \\
(\mathrm{M} \pm \mathrm{SD})\end{array}$ & $\begin{array}{l}\mathrm{MCS} \\
(\mathrm{M} \pm \mathrm{SD})\end{array}$ & $p$ \\
\hline Wound size (cm) & $8.4 \pm 1.45$ & $3.1 \pm 0.42$ & $<0.001$ \\
OP time (min) & $43 \pm 9.6$ & $40 \pm 5.8$ & 0.37 \\
Haemoglobin drop (g/L) & $28.6 \pm 3.5$ & $15.4 \pm 2.1$ & $<0.001$ \\
Hospital stay (days) & $10.6 \pm 1.92$ & $6.2 \pm 1.52$ & $<0.001$ \\
Blood transfusion & $10 / 40$ & $2 / 44$ & 0.008 \\
Adequate reduction & $40 / 40$ & $44 / 44$ & 1.0 \\
Adequate screw position & $36 / 40$ & $34 / 44$ & 0.15 \\
Healing time (days) & $85.8 \pm 17.2$ & $91.7 \pm 15.9$ & 0.28 \\
\hline
\end{tabular}

DHS=dynamic hip screw

MCS $=$ multiple cannulated screws

$\mathrm{M}=$ Mean

$\mathrm{SD}=$ Standard Deviation

We defined overall success as stable fixation without need to change the implant. Overall failure was defined as having to change the implant, by the last clinical follow-up, due to implant failure, symptomatic non-union or a severe avascular necrosis (AVN). Although a mild AVN without exchanging the implant at the last follow-up did not belong to failure, it should be regarded as a complication. In the DHS group, the implant was deemed to have failed mechanically if the lag screw migrated within or cut out of the femoral head, the side plate was bent or broken, or the cortical screws loosened. In the DHS group, no patient $(0 \%)$ showed evidence of mechanical failure and none $(0 \%)$ developed non-union (Fig. 1). However, three of 40 patients $(7.5 \%)$ had AVN changes in the femoral head (Ficat stage II) but all were still pain free at the final followup. Another patient (2.5\%) had severe AVN (Ficat stage III) associated with painful daily activity and underwent hemiarthroplasty. In this case, the primary surgery was delayed due to missed diagnosis for nine days. In total, only one DHS patient $(2.5 \%)$ required change of implant and the overall success rate was $97.5 \%$. In the MCS group, three patients (6.8\%) had implant failures due to early screw loosening with displaced fractured gap. In these cases, all had adequate screw positions initially. Hemiarthroplasty was performed on these patients. Another two patients (4.5\%) had non-union and underwent arthroplasty. Two cases had an inadequate screw position. A further two patients (4.5\%) showed mild AVN changes (Ficat stage II) and were treated conservatively. Another two patients (4.5\%) developed severe AVN (Ficat stage III) and underwent hemiarthroplasty. One of the above-mentioned two patients was diabetic with chronic renal insufficiency (Fig. 2). In total, seven patients $(15.9 \%)$ in the MCS group required change of implants at the last follow-up, and the overall success rate was $84.1 \%$. We concluded that the total complications were no different between the DHS and MCS groups 
Fig. 1 A 78-year-old male patient with femoral neck fracture was treated with dynamic hip screw. (a) Preoperative radiograph showed an undisplaced intracapsular femoral neck fracture. (b) Radiograph at the final follow-up (postoperatively 30 months) showed solid union without any evidence of mechanical failure
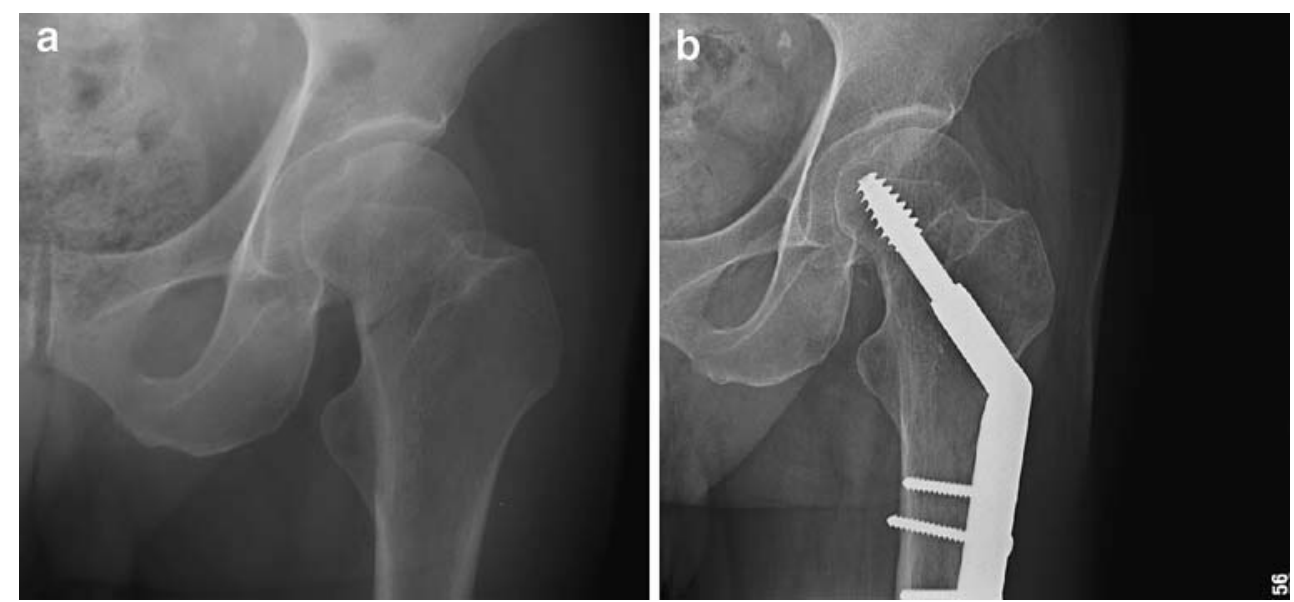

( $10 \%$ vs. $20.5 \%, p=0.24)$. However, the DHS group showed a significantly increased rate of overall success compared with the MCS group ( $97.5 \%$ vs. $84.1 \%, p=0.04$ ) (Table 3).

At the final follow-up, the Harris hip scores of the patients were evaluated for the functional outcomes. Differences in mean hip score between the two groups were not significant $(p=0.65)$. The mean hip score was $84.2 \pm 5.2$ points for DHS group and $82.6 \pm 5.1$ points for MCS group.

\section{Discussion}

Previously, MCS or DHS have been commonly used to treat elderly patients with undisplaced femoral neck fractures [6, 10, 12, 14]. However, comparison between the two devices has been rare. Clark et al. [7] determined that there was no significant difference between the fixation afforded by the DHS and MCS in five pairs of female cadaver femora. They concluded that bone quality played an important role in the stability of the bone implant unit. In our study, the Singh index was used to define bone quality and showed no difference between the
DHS and MCS groups. Particularly, the DHS group showed no evidence of mechanical failure. In contrast, the MCS group had three implant failures due to early screw loosening with displaced fracture gap that might be related to lateral cortex osteoporosis found in elderly patients (Singh index: 2, 2 and 3, respectively). We felt that the fixation power of MCS might be similar to DHS initially. However, for an elderly patient, MCS fixation could loosen with time if the lateral cortex was osteopenic with poor bone quality.

In this study, although 3-hole DHS fixation for an elderly patient with a femoral neck fracture achieved a higher overall success rate, the larger incision with more soft tissue dissection and more blood loss were considered as disadvantages. Many authors have attempted to find less invasive DHS with a shorter side plate for treating proximal femoral fractures $[1,3,16]$. However, the adequate length of side plate is still controversial. Heyse-Moore [12] reported fixation of intracapsular femoral neck fractures with a one-hole plate DHS. However, the shorter side plate with only one cortical screw fixation may loosen if the femoral cortex is osteoporotic. Although several authors have reported the use of a 2 -hole side plate that produced
Fig. 2 A 75-year-old female patient with chronic renal insufficiency had an undisplaced femoral neck fracture treated with three cannulated screws. (a) Preoperative radiograph showed undisplaced femoral neck fracture (arrow). (b) Radiograph at postoperatively 4 months showed severe avascular necrosis of femoral head
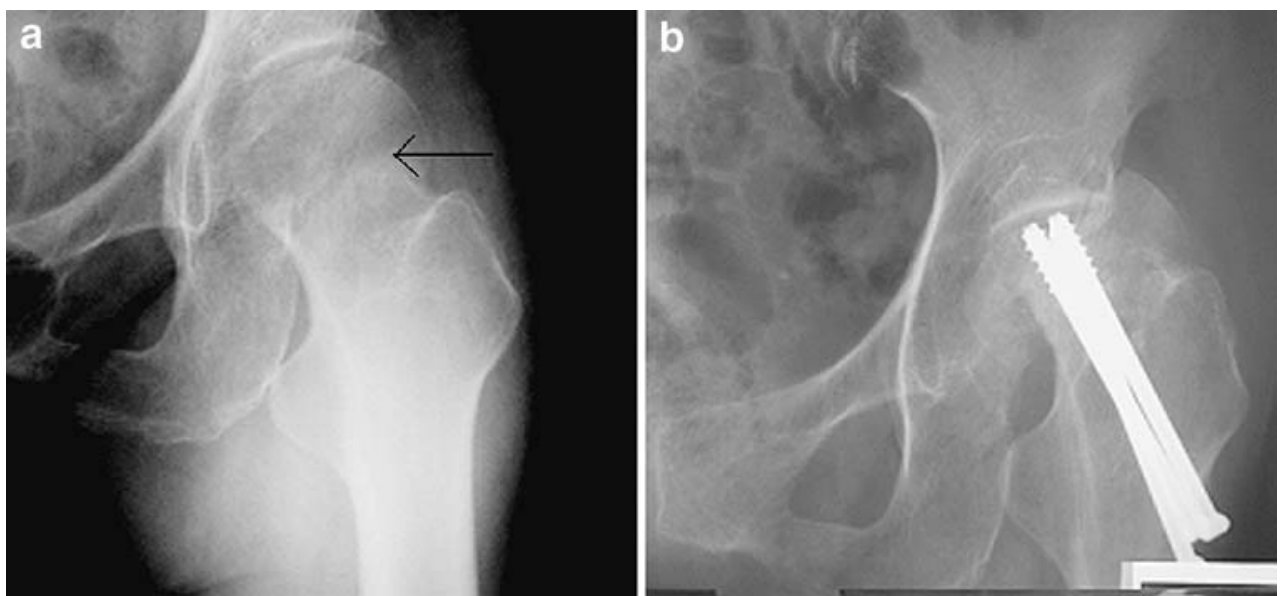
Table 3 Complications and failures in the two groups

\begin{tabular}{llll}
\hline & DHS & MCS & $p$ value \\
\hline Implant failure (N) & 0 & $3(6.8 \%)$ & 0.24 \\
Non-union (N) & 0 & $2(4.5 \%)$ & 0.50 \\
Severe AVN (N) & $1(2.5 \%)$ & $2(4.5 \%)$ & 1.0 \\
Mild AVN (N) & $3(7.5 \%)$ & $2(4.5 \%)$ & 0.67 \\
Total complication (N) & $4(10 \%)$ & $9(20.5 \%)$ & 0.24 \\
Overall Failure (N) & $1(2.5 \%)$ & $7(15.9 \%)$ & 0.04 \\
Overall success (N) & $39(97.5 \%)$ & $37(84.1 \%)$ & 0.04 \\
\hline
\end{tabular}

$\mathrm{N}=$ number of patients

satisfactory healing, to date, the 2-hole DHS has not been widely used in our hospital.

A device that allows fracture impaction can decrease the rate of delayed union or non-union. The DHS has effective intra-operative compression of the fracture site but its use alone has not previously been recommended as poor fixation and loss of reduction may occur due to lack of control of the proximal fragment, which may rotate during insertion of the lag screw. Rau et al. [18] reported this complication in $20 \%$ of their cases. In our study, DHS for undisplaced femoral neck fractures had a $100 \%$ union rate without any complications associated with proximal fragment rotation, intra- or post-operation. Several reasons were related to our high union and low complication rate: (a) the marked compressive effect of DHS increased fracture healing and resistance to postoperative fragment spinning; (b) the temporary use of a secondary guide wire before lag screw insertion enabled control of proximal fragment rotation; (c) the DHS procedure was simple associated with a high rate of adequate screw position. Previous studies have shown that the adequate MCS position can enhance a compressive effect of the fracture site and afford better stability of fixation [4, 6, 21]. However, the standard procedure for MCS fixation is technically demanding [6]. Lagerby et al. [15] reported that the adequate MCS position was only $56.3 \%$ (151/268). In our study, the MCS showed a trend of a decreased rate of adequate screw position compared with DHS. Furthermore, there was a nonunion in the MCS group that might be related to an inadequate screw position.

Brodetti [5] reported that a large lag screw placed suboptimally could damage the blood supply of the femoral head and result in late AVN. However, in our study, the AVN rate was no different between the two fixation methods. There were 4/40 (10\%) of large lag screw (DHS) and 4/44 (9.1\%) of small lag screw (MCS) developing AVN changes. Further, one case with a prolonged injury-surgery interval, and one case with a poor medical condition developed AVN. Therefore, we concluded that the causes of late AVN after union must be multifactorial.

This study had various limitations: (a) it was performed in a teaching institution where the resident was the operating surgeon and the surgeons' level of experience could influence the outcomes; (b) Singh index was used to evaluate bone quality but studies of interobserver reliability show that there are difficulties in differentiating the six types with this method; (c) the study was not a large series and when we calculated the power of this study, most were only $85 \%-90 \%$. If the null hypothesis (no difference between the two groups) was accepted in our study, the false-negative rate was up to $10-15 \%$.

In conclusion, although DHS fixation has larger skin incision and more soft tissue dissection, its use in elderly patients with osteoporosis is still recommended due to simplicity, efficacy and high overall success rate.

\section{References}

1. Alobaid A, Harry EJ, Elder GM et al (2004) Minimally invasive dynamic hip screw: prospective randomized trial of two techniques of insertion of a standard dynamic fixation device. J Orthop Trauma 18:207-212

2. Asnis SE, Wanek-Sgaglione L (1994) Intracapsular fractures of the femoral neck. Results of cannulated screw fixation. J Bone Joint Surg Am 76(12):1793-1803

3. Bolhofner B, Russo P, Carmen B (1999) Results of intertrochanteric femur fractures treated with a 135-degree sliding screw with a two-hole side plate. J Orthop Trauma 13:5-8

4. Bray TJ (1997) Femoral neck fracture fixation, clinical decision making. Clin Orthop 339:20-31

5. Brodetti A (1960) The blood supply to the femoral neck and head in relation to the damaging effecting effects of nails and screws. J Bone Joint Surg Br 42:794-801

6. Chen WC, Yu SW, Tseng IC et al (2005) Treatment of undisplaced femoral neck fractures in the elderly. J Trauma 58:1035-1039

7. Clark DI, Crofts CE, Saleh M (1990) Femoral neck fracture fixation. Comparison of a sliding screw with lag screws. J Bone Joint Surg Br 72:797-800

8. Cserhati P, Kazar G, Manninger J et al (1996) Non-operative or operative treatment for undisplaced femoral neck fractures. A comparative study of 122 non-operative and 125 operatively treated cases. Injury 27:583-588

9. DeLee JC (1991) Fractures and dislocations of the hip. In Rockwood CA Jr, Green DP, Bucholz RW (eds) Rockwood and Green's fractures in adult. 3rd edn. Philadelphia, PA: LippincottRaven: 1481-1651

10. Eisler J, Cornwall R, Strauss E et al (2002) Outcomes of elderly patients with nondisplaced femoral neck fractures. Clin Orthop 399:52-58

11. Heetveld MJ, Raaymakers ELFB, van Walsum ADP et al (2005) Observer assessment of femoral neck radiographs after reduction and dynamic hip screw fixation. Arch Orthop Trauma Surg 125:160-165

12. Heyse-Moore GH (1996) Fixation of intracapsular femoral neck fractures with a one-hole plate dynamic hip screw. Injury 27:181183

13. Johansson T, Jacobsson SA, Iverson I et al (2000) Internal fixation versus total hip arthroplasty in the treatment of displaced femoral neck fractures: a prospective randomized study of 100 hips. Acta Orthop Scand 71:597-602

14. Kuokkanen H, Korkala O, Antti-Poika et al (1991) Three cancellous bone screw versus a screw-angle plate in the treatment 
of Garden I and II fractures of the femoral neck. Acta Orthop Belg 57:53-57

15. Lagerby M, Asplund S, Ringqvist I (1998) Cannulated screws for fixation of femoral neck fractures. Acta Orthop Scand 69:387-391

16. McLoughlin S, Wheeler D, Rider J et al (2000) Biomechanical evaluation of the dynamic hip screw with two- and four-hole side plates. J Orthop Trauma 14:318-323

17. Ort PJ, LaMont J (1984) Treatment of femoral neck fractures with a sliding compression screw and two Knowles pins. Clin Orthop 190:158-162

18. Rau FD, Manoli A, Morawa LG (1982) Treatment of femoral neck fracture with the sliding compression screw. Clin Orthop 163:137-140

19. Rehnberg L, Olerud C (1989) Subchondral screw fixation for femoral neck fractures. J Bone Joint Surg Br 71:178-180
20. Rogmark C, Carlsson A, Johnell O et al (2002) A prospective randomized trial of internal fixation versus arthroplasty for displaced fractures of the neck of femur. J Bone Joint Surg Br 84:183-188

21. Selvan VT, Oakley MJ, Rangan A et al (2004) Optimum configuration of cannulated hip screws for the fixation of intracapsular hip fractures: a biomechanical study. Injury 35:136-141

22. Singh M, Nagrath AR, Maini PS (1970) Changes in trabecular pattern of the upper end of the femur as an index of osteoporosis. J Bone Joint Surg Am 52:457-467

23. Skinner PW, Powles D (1986) Compression screw fixation for displaced subcapital fracture of the femur. Success or failure? J Bone Joint Surg Br 68:78-82

24. Sorensen JL, Varmarken JE, Bomler J (1992) Internal fixation of femoral neck fractures. Dynamic hip and Gouffon screws compared in 73 patients. Acta Orthop Scand 63:288-292 